\title{
MAPPING WITH A SINGLE-SPACING INTERFEROMETER
}

\author{
B. A. Doubinsky \\ Institute of Radioengineering and Electronics, U.S.S.R. Academy of Sciences \\ Moscow, U.S.S.R. \\ N. S. Kardashev \\ Space Research Institute, U.S.S.R. Acaderny of Sciences \\ Moscow, U.S.S.R.
}

Recent VLBI development provides opportunities for aperture synthesis, or super synthesis, with terrestrial interferometer antennas, and also in the near future with orbiting antennas. In this connection it is useful to consider the potential mapping capability of a single interferometer. Usually one would assume the use of image restoration procedures to be indispensable for observations with a single interferometer, because the $u v$-plane is covered only on an elliptical track, and is very much unfilled. High and distant sidelobes of the synthesized beam, caused by gaps in the coverage of the $u v$-plane, are usually considered to be influenced only to a small degree by any weighting function by which the visibility function is multiplied in the Fourier image formation algorithm. It has been shown that the mean-squared value of the point-source response function is invariant with respect to the weighting function (Doubinsky 1983). However, this does not prohibit tha modulation of the point-source response by the visibility weighting function. This study shows that one can suppress the sidelobes in two symmetrical sectors of the point-source response with the help of such modulation. Thus for a lineshaped source model (that is, linear within the angular resolution of the synthesized beam) one could map without excessive distortion by Fourier transformation alone (Doubinsky 1979). The exclusion of restoration procedures, which are largely empirical, increases the reliability of the image when the source structure is extended and the signal-to-noise ratio is low. It also saves much computer time.

The linear form of the model is a significant restriction. Nevertheless, in radio astronomy there are many objects that appear to be linear: for example, a radio galaxy with fairly compact radio emitting regions symmetrically located on each side. Many unresolved sources may be shown to be of similar type by future VLBI observations. Such a one-dimensional image can be analyzed as a strip brightness distribution when the width of the $u v$-track in a direction orthogonal to the long axis of the source is small enough so that the source is not resolved in that direction.

As a simple illustration of the principles involved, consider a source that is linear in the east-west direction. Then the visibility is a function only of $u$, and in the $v$ direction remains constant out to values beyond the projected spacings of the interferometer. If the source is observed continuously over a sufficient portion of the $u v$-track, the visibility is determined continuously as a function of $u$ from zero to the maximum spacing in the $u$ direction, with no gaps in the $u$-coverage. Then if the source is also well resolved by the maximum spacing in the $u$ direction, the eastwest profile can be accurately determined. The point-source response function, 
with which the source profile is convolved, can be controlled by introducing a visibility weighting function equal to the Fourier transform of the desired pointsource response. The same considerations can be applied to a linear source at any position angle by appropriate rotation of axes. The linear-model analysis has been applied to the source $3 \mathrm{C263}$, in which four components are widely spaced in a linear manner. The visibility data used were obtained at the Mullard Radio Astronomy Observatory, and the brightness profile derived is in strict agreement with the two-dimensional map of the source.

The more general case of a curvilinear source has also been considered. If the minimum radius of curvature of such a source is large compared with the width of the synthesized beam, then the source can be treated as a number of linear components.

In the case where the form of the source is initially unknown, a twodimensional map can first be made by two-dimensional Fourier transformation of the visibility data. The resulting beam will contain large sidelobes, but the map should reveal whether any major component of the source is of linear form, and if such a component exists, the position angle can be estimated. The linear-model analysis can than be applied as a second step.

In conclusion, the authors have demonstrated a simplified approach to the analysis of interferometer observations of sources of linear form. This work has been stimulated by the prospect of increased synthesis mapping with the use of both terrestrial and space-based VLBI antennas. The authors wish to express their thanks to Dr. G. G. Pooley who kindly provided the data for $3 \mathrm{C} 263$ for two different interferometer baselines.

\section{REFERENCES}

Doubinsky, B. A. Astronom. Letters, 1979, 5(10), 557-560 (in Russian).

Doubinsky, B. A. Radiophysics, 1983, 26(11), 1493-1496 (in Russian). 